\title{
Natural killer cells and immune surveillance
}

\author{
Mariana Jobim, ${ }^{1}$ Luiz F. J. Jobim²
}

\begin{abstract}
Objectives: To analyze the importance of natural killer cells, their killer immunoglobulin-like receptors (KIR) and genes in autoimmunity and in the immune surveillance against infectious agents and stem cells transplantation. The characteristics and polymorphisms of the KIR genes and receptors in the Brazilian population is described.
\end{abstract}

Sources: Textbooks, review articles and recent scientific articles are cited and listed in the references.

Summary of the findings: KIR genes and haplotypes within a Brazilian Caucasian population were surveyed and analyzed to assess the future relationship of this system with diseases. Of 116 voluntary bone marrow donors, we identified 32 genotypes with frequencies of A and B haplotypes of 51 and $49 \%$, respectively. A comparative analysis was performed between these genotypes and those from other populations.

Conclusions: Innate immunity is an important anti-infectious barrier in newborns. It is independent of both cellular and humoral immunity, can be faster and confers great advantage in early age. At the same time, it stimulates CD8 T lymphocytes to act and amplify the immunological protection network. Nevertheless, as in the majority of situations in which immunity is activated, it can also be harmful, damaging the body through autoimmune mechanisms or even, through its absence, creating space for infectious agents to act free. Our study of a control group for KIR genotype and haplotypes in Brazilian Caucasoids could be used in future analyse of diseases related to these genes.

J Pediatr (Rio J). 2008;84(4 Suppl):S58-67: KIR genes, natural killer cells, KIR genotypes in Brazilian Caucasoids.

\section{Introduction}

The immune system is a network of cellular and humoral components with the function of "self/non-self" discrimination. When it works well, it can destroy infectious agents, tumor cells and non identical transplanted cells.

Natural killer cells (NK) are an important part of this immune surveillance. They account for around 10 to $20 \%$ of circulating lymphocytes. Morphologically, they are larger than Tand B lymphocytes, exhibiting granular cytoplasm and CD16 and CD56 surface markers. They differ from the members of the adaptive immunity system by a fast reaction, sometimes in few hours, during invasion by viruses or bacteria. In contrast, T lymphocytes can take days to initiate an effective immune response, even though they do have much in common with NK cells, especially in terms of surface markers, cytotoxic effectors function mediated by secretion of cytokines, perforins, granzymes and interferon $y$ (INF- $Y$ ) and a close relationship with dendritic cells (DC). Natural killer cells exhibit certain differences, such as absence of the T cell receptor, which is the main molecule in T-lymphocyte immune response. The latter must pass through the thymus to enter the circulation to be effective in immune surveillance. ${ }^{1}$ The NK cells are thymus-independent, and patients with NK deficiencies suffer from persistent viral infections, particularly caused by herpes viruses. In such patients, these agents can only be eliminated using antiviral drugs, even though they may have adaptive immunoresponse. ${ }^{2}$

When NK cells are isolated from blood they are capable of destroying certain types of target-cell. This baseline level increase by 20 to 100 times in response to exposure to interferon a (INF-a), interferon $\beta$ (INF- $\beta$ ) and IL-12 produced by macrophages in response to viral agents. These lymphokines can activate NK cells to destroy infectious agents immediately or when CD8 T lymphocytes initiate their activities.

1. Mestre, Universidade Federal do Rio Grande do Sul (UFRGS), Porto Alegre, RS, Brazil. Médica, Serviço de Imunologia, Hospital de Clínicas de Porto Alegre (HCPA), Porto Alegre, RS, Brazil.

2. Doutor. Professor associado, Faculdade de Medicina, UFRGS, Porto Alegre, RS, Brazil. Chefe, Serviço de Imunologia, HCPA, Porto Alegre, RS, Brazil.

No conflicts of interest declared concerning the publication of this article.

Suggested citation: Jobim M, Jobim LF. Natural killer cells and immune surveillance. J Pediatr (Rio J). 2008;84(4 Suppl):S58-67.

doi:10.2223/JPED.1780 
Interferon- $\alpha$ and INF- $\beta$ promote the cytotoxic effects of NK cells, while IL-12 stimulates cytokine production, including INF- $\gamma$, creating a positive feedback process of both types of cells in the infected tissue. The manifestation of this is that NK cells predominate during the onset of infections, with relation to INF-Y production, activating the macrophages to secrete lymphokines which in turn start the adaptive immune response with the arrival of $\mathrm{T}$ Iymphocytes. When they start to work, NK cells may reduce activity in response to production of IL- 10 by T lymphocytes. ${ }^{2}$

Natural killer cells have a large number of surface receptors, which are also present in certain T-lymphocyte and are responsible for the identification of infectious agents and abnormal cells. The class I major histocompatibility complex molecules, participate in this process by their human leukocyte antigen (HLA), important molecules in understanding the function of NK cells. ${ }^{3}$ These proteins are also of great importance in the recognition of antigens by $\mathrm{T}$ Iymphocytes, although in a different manner from what happens with the NK cells. The T lymphocytes recognize antigenic peptides that are exposed on the surface of HLA molecules in antigen presenting cells by the $T$ cell receptors. After this, lymphocytes are active, producing lymphokines and sending stimulatory signals to other immune system cells. In contrast, the NK cells recognize infected, abnormal or transplanted cells by the "absence or presence" of HLA molecules on their surfaces. Therefore, we can understand the HLA system as being the helper of two important "masters" in defense and immune surveillance: the NK cells of innate immunoresponse and the cytolytic T lymphocytes of adaptive immunoresponse.

At this point we should mention the "missing-self recognition" hypothesis, i.e., the recognition of reduced or even absent expression of HLA molecules in infected cells and in tumor cells or recognition of transplanted cells with foreign or different HLA molecules, activating the NK cells which become aggressive and potentially destructive. ${ }^{4,5}$ Therefore, the NK cells' role in immune surveillance is dependent on appropriate and specific concentrations of HLA molecules on the surfaces of other target-cells. In contrast, normal cells which have normal levels of identical HLA molecules on their surfaces are recognized by NK cells which generate an inhibition signal, preventing their destruction. ${ }^{6}$

The NK cells can be activated and become cytotoxic as a result of exaggerated expression of ligands for activation receptors on the target-cell-surface. ${ }^{5}$ In this manner, NK cells can perform immune surveillance both by recognition of reduced or absent expression of HLA molecules on target-cells and in response to imbalances between activation and inhibition signals mediated by receptors in the NK cells, known as killer immunoglobulin-like receptors (KIR). These protein receptors are important in the destruction of abnormal cells and are the expression of a cluster of genes identified as the KIR genes.

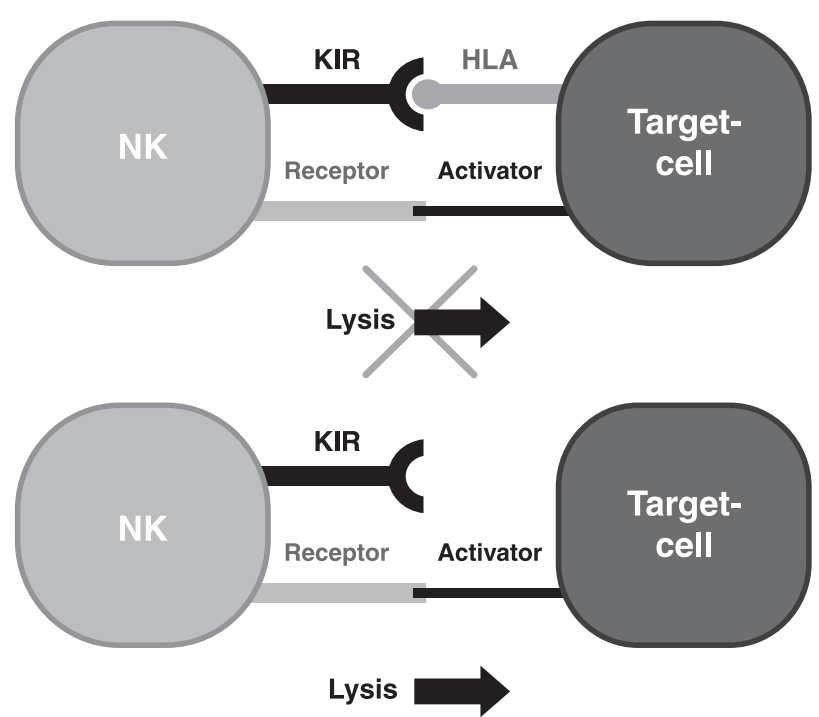

HLA = human leukocyte antigen; KIR = killer immunoglobulin-like receptor; NK $=$ natural killer cell.

Figure 1 - Relationships between NK cell and target cell, depending on the presence or absence of HLA ligands

Innate immunity is of great importance in pediatrics. Many infectious diseases can be effectively neutralized even before the adaptive immune system has started. In certain diseases with abnormal or low expression on the cell surface - such as on viral infected and on tumors cells - the absence of an inhibition signal, in addition to preventing inhibition itself, allows activation of the NK cells. ${ }^{7,8}$ On the other hand, there are also autoimmune diseases that are influenced by NK cell activation and inhibition, and some patients can be protected or not by the innate system after receiving bone marrow transplantation (BMT) to treat leukemia (Figure 1).

\section{Nomenclature and polymorphisms of KIR genes and receptors}

Natural killer cells circulate in the blood in a practically activated state, ready to attack infected tissues as soon as macrophages sound the alarm. The NK cells are maintained in this state by a system of receptors which release activation or inhibition signals. ${ }^{8}$

The nomenclature of NK cell receptors is based on their extracellular and intracellular structure (Figure 2). These molecules are similar to immunoglobulins with two or three extracellular domains (2D and $3 \mathrm{D}$ ) used to bind to polymorphic determinants of the HLA-A, B and C systems, a transmembrane (TM) region and a cytoplasmic tail (CT), which may be short (S) or long ( $L$ ). The names used for the different KIRs and the sequences of their respective genes and alleles can be accessed on the KIR database (http://www.ebi.ac.uk/ipd/kir). ${ }^{9}$

The CT and TM regions control functional activity. Molecules with short tails (S) are activating, while long tails (L) 


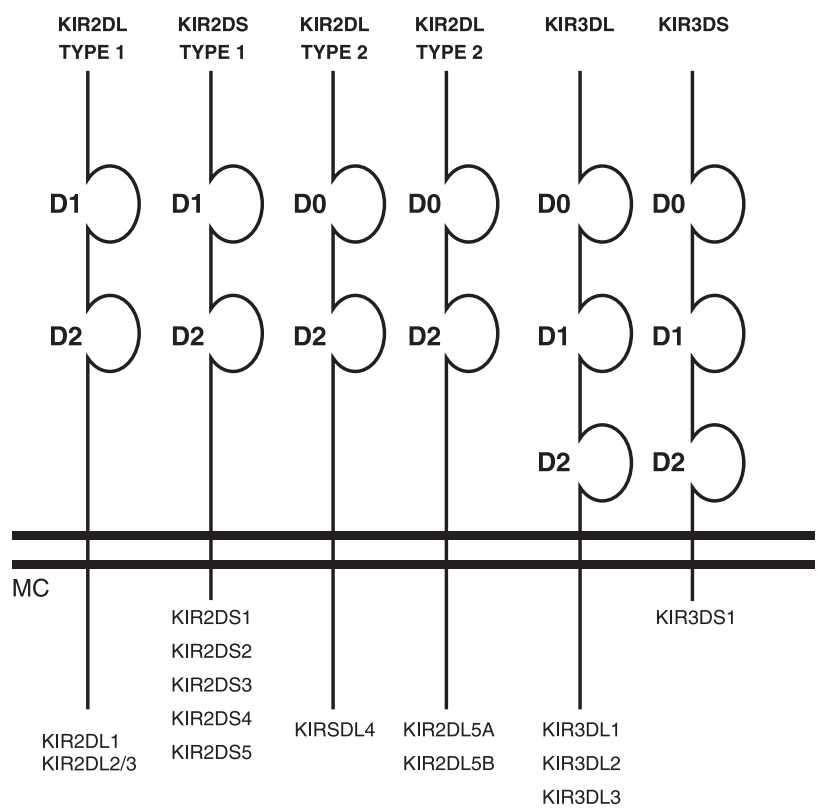

Figure 2 - KIR receptors in the cellular membrane (CM). The KIR have two or three extracellular domains (D0, D1 and D2), and either short (S) or long (L) cytoplasmic tails, which define whether the molecule is activating $(\mathrm{S})$ or inhibitory (L)

are inhibitory (with the exception of KIR2DL4). Molecules with the long tail have one or two immunoreceptors with an immune tyrosine-based inhibitory motif (ITIM). In contrast, short tail receptors do not have ITIM, but a positively-charged amino acid in the TM domain, wich allows interaction with the DAP-12 accessory molecule, releasing an activation signal via an immunoreceptor tyrosine-based activation motif (ITAM). Therefore, the KIR2DS1 receptor is a molecule with two extracellular domains and a single CT - short and activating.

The KIR gene family consists of fifteen genes (KIR2DL1, KIR2DL2，KIR2DL3，KIR2DL4，KIR2DL5A，KIR2DL5B， KIR2DS1, KIR2DS2, KIR2DS3, KIR2DS4, KIR2DS5, KIR3DL1, KIR3DL2, KIR3DL3 and KIR3DS1), and two pseudogenes (KIR2DP1 and KIR3DP1), located in a region around $150 \mathrm{~Kb}$ from the leukocyte receptor complex (LRC) on chromosome 19q13.4. The different KIR genes exhibit great molecular similarity with each other and have originated from one ancestral gene by a series of duplications, recombinations and mutations. The basic KIR gene structure is a nine-exon unit that represents the ancestral gene. ${ }^{10}$

More than 100 different genotypes were detected by an international collaborative study. ${ }^{10}$ We found 32 genotypes in 116 Brazilian Caucasoids, the two most common of which have frequencies of 24 and $13.80 \%$ (Figure 3 ). The same genotypes were also the most prevalent in other studies. ${ }^{11}$

The KIR genes are inherited in blocks, or haplotypes, as are the HLA genes. Although there are a large number of KIR haplotypes, some are more prevalent, including the $A$ and $B$ haplotypes (Figure 4). The A haplotype has seven loci and its major characteristic is increased expression of inhibitory
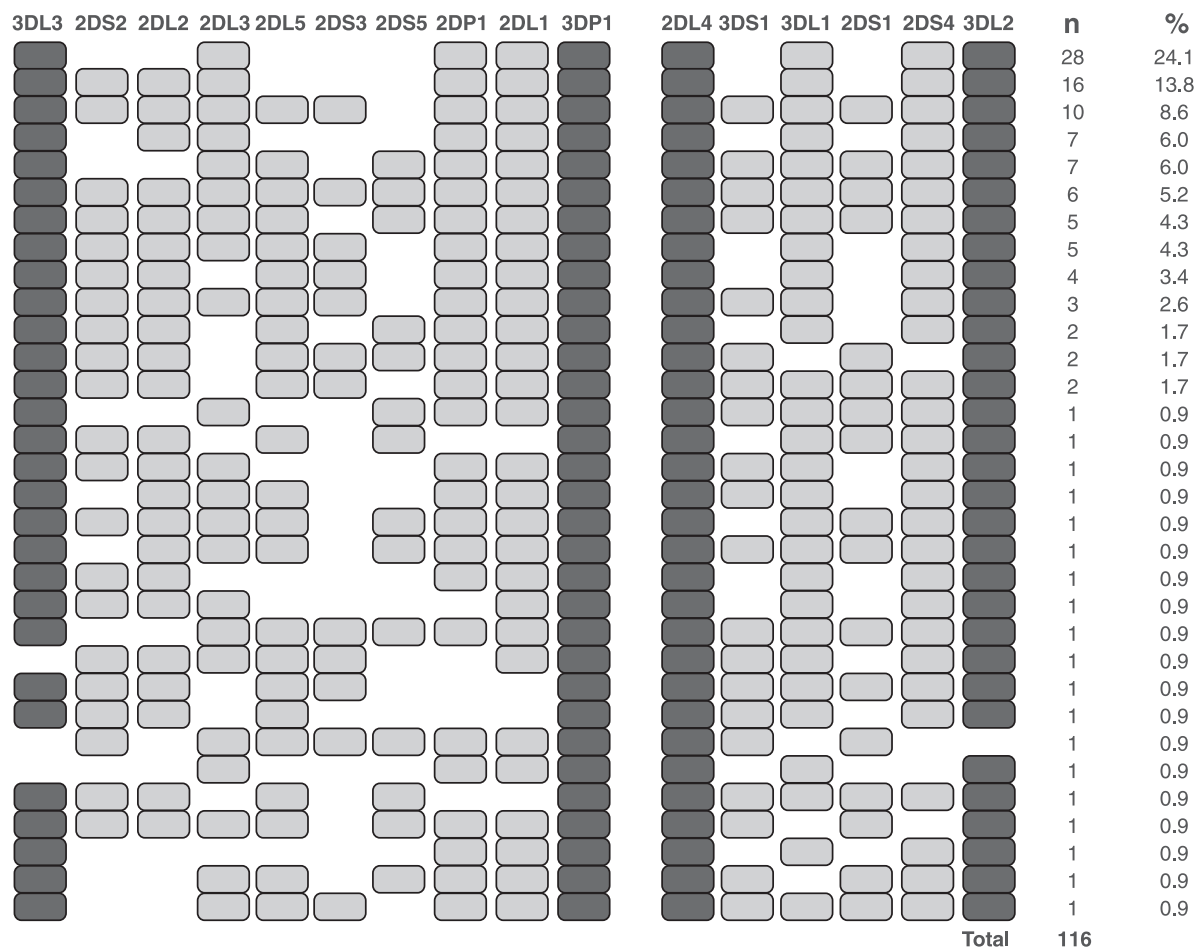

Figure 3 - KIR Genes: haplotypes A and B 


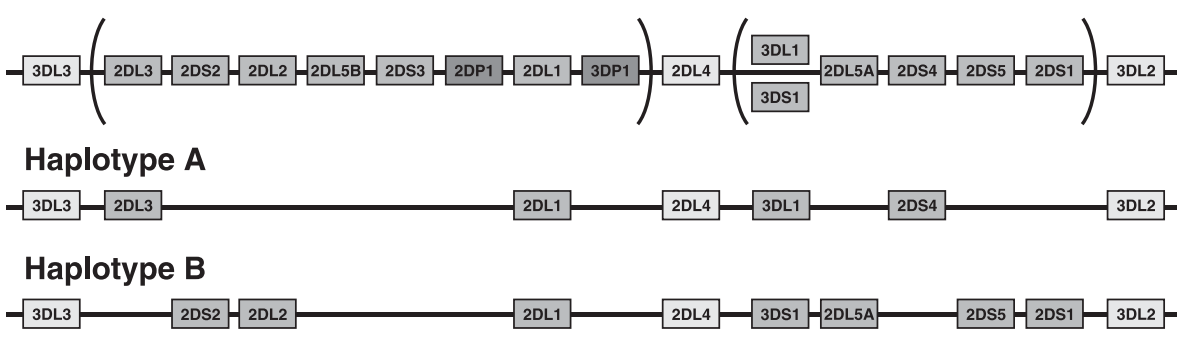

Figure 4 - KIR Genes: haplotypes A and B

genes or receptors. It is defined by the presence of KIR2DL1, KIR, KIR, KIR, KIR and KIR. The only stimulatory receptor in the A haplotype is KIR2DS4. The $B$ haplotype has several combinations of KIR, KIR, KIR, KIR, KIR and KIR. The B haplotype has more activating genes, and is defined by the presence of KIR2DL2 and absence of KIR3DL1 and KIR2DL3. ${ }^{12,13}$ The genes KIR and KIR are present in both haplotypes, and are known as structural or framework genes. In certain haplotypes, we may observe the absence of one of the framework genes. ${ }^{14}$

Studies suggest that NK cells do not react against other cells in the body under normal conditions. Therefore, NK cells that do not express inhibitory receptors that recognize self-HLA are unresponsive to and relatively tolerant to autologous cells. ${ }^{14}$ Expression of KIR is restricted to NK cells and certain T cells, known as NKT.

\section{Natural killer cell receptor ligands}

The ligands for KIR receptors are the class I HLA molecules (HLA-A, B and C). The receptors KIRD2DL2, KIRD2DL3 and KIRD2DS3 recognize certain HLA-C molecules, identified as members of group 1 (HLA-C1). In counterpoint, the receptors KIR2DL1 and KIR2DS1 recognize group 2 (HLA-C2) (Table 1). Groups 1 and 2 are distinguished by a dimorphism at position 80 of helix of the HLA-C molecule. ${ }^{15}$ Both forms are characterized by the presence of Ser77/Asn80 and Asn77/Lys80, and there is a certain hierarchy to the interaction of KIR with HLA-C molecules, been the interaction KIR2DL1-C2 stronger than KIR2DL3-C1.

After a BMT, the donor's NK cells that express only receptors for group C2 may lyse the patient's group C1 homozygous target-cells. The same can happen if the donor is homozygous for $\mathrm{C} 2$, and the receptor, homozygous for $\mathrm{C} 1$.
The HLA-C, the HLA-B locus can also be divided into two groups: Bw4 and Bw6. The KIR3DL1 interacts with HLA-B molecules if they have Bw4 serology, and, if these have isoleucine (Ile) in position 80 , there is greater inhibition of NK-mediated lysis. There are no known high affinity interaction with Bw6 molecules. ${ }^{16}$ The KIR2DL4 molecule binds to HLA-G, which is a non-classical HLA type with little polymorphism and which is expressed in endothelial cells of the thymus, fetal trophoblasts and cornea. Resting NK cells can be stimulated to produce cytokines and chemokines by soluble HLA-G. The KIR2DL4 gene is a framework gene found in almost all individuals, although in around $50 \%$ of people it is not expressed, suggesting that this gene could be subjected to some type of selection. Therefore, if we assume that the KIR2DL4-HLA-G interaction is physiologically relevant, it is also possible to suppose that the presence of alleles that do not express it is due to some type of disadvantage under certain conditions.

It is important to point out that interactions between KIR and its HLA ligands can be modified by peptides located in the $T$-cell receptor molecule binding region. Of interest is the interaction between KIR3DL2 and HLA-A3 and A11.

\section{Laboratory identification of the KIR genes}

Blood samples were collected to determine the distribution of the KIR genotypes and haplotypes in a Brazilian Caucasoid population. The samples were collected in EDTA and DNA extracted using the salting-out procedure. ${ }^{17}$ The DNA samples were analyzed using polymerase chain reaction with specific primers (PCR-SSP) for 15 KIR genes.

The PCR primer sequences were based on earlier publications. ${ }^{18}$ The amplification products were viewed on a transilluminator with ultraviolet light, after electrophoresis in

Table 1 - KIR receptors and their HLA-C-specific ligands

\begin{tabular}{lc}
\hline KIR receptor & Specific ligand \\
\hline KIR2DL2, KIR2DL3, KIR2DS2 & Group 1 (HLA CW 1, 3, 7,8) \\
KIR2DL1 and KIR2DS1 & Group 2 (HLA CW 2, 4,5, 6) \\
KIR3DL2 & HLA-A03, A11 \\
KIR2DL4 & HLA-G
\end{tabular}




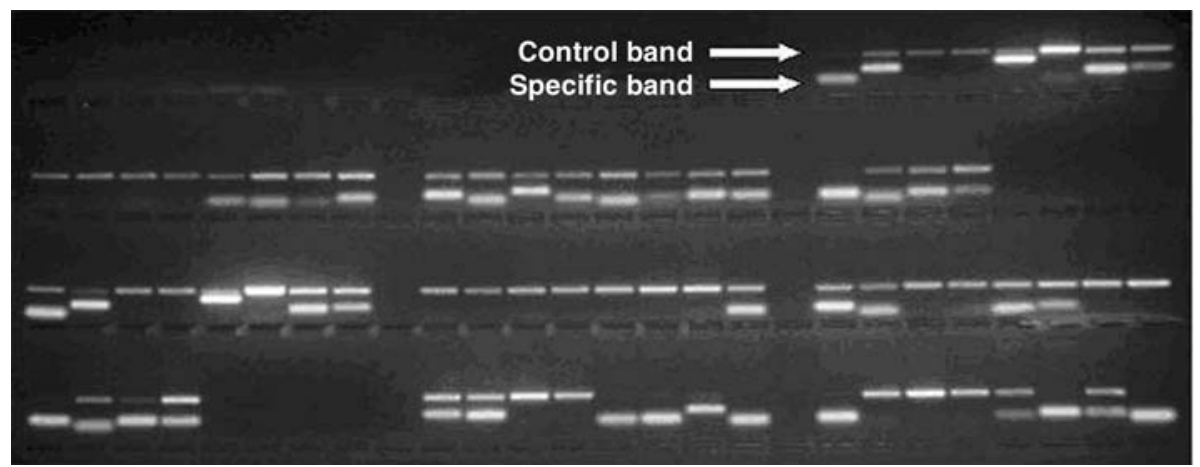

Figure 5 - Typing of KIR genes by polymerase chain reaction with specific primers (PCR-SSP). The control band, positive in almost all positions, identifies the internal control. The specific band identifies each KIR allele by transillumination in agarose gel with ethidium bromide, according with the expected results for the location and the size of the band

agarose gel at $1 \%$, containing ethidium bromide and typed according to their size. A sample of such assay is shown in Figure 5.

The KIR genes were also typed by sequence-specific oligonucleotides (PCR-SSO) using LabtypeSSO ${ }^{\circledR}$ reagents (Teppnel-United States).

Alleles of the HLA-Cw system were also determined using PCR-SSP. ${ }^{19}$

Results obtained with the two methods (PCR-SSO and PCR-SSP) for the 116 blood samples of the mentioned Brazilian Caucasoid population were absolutely identical, with no differences between both methods (results not shown).

\section{Comparison of KIR genes of a Brazilian Caucasian population with other populations}

The frequency of the KIR genes of the 116 Brazilian Caucasoid was compared with those of other populations. ${ }^{20-25}$ The Brazilian, English and Argentine Caucasian populations exhibit similar frequencies of the KIR genes (Table 2). Significant differences exist between the indigenous populations of Argentina and Mexico, when compared with the Caucasoid populations. The KIR2DS3 activator gene in particular is different, since, either they do not have this allele, or they have it in low frequency, in common with certain oriental populations (Chinese and Japanese). The same KIR2DS3 have an intermediate frequency in the Caucasoid populations, in contrast with $81 \%$ observed among Australian aboriginal populations (Table 2). There are other differences in relation to other populations, demonstrating the different distribution of polymorphism for these markers and predicting differences in innate immune response between populations.

Among Caucasoids, the A and $B$ haplotypes are distributed similarly. ${ }^{12,13}$ Of the 116 Brazilian Caucasoid donors, we found 51 and $49 \%$ of the $A$ and $B$ haplotypes, respectively.

One feature of interest is the difference in the frequency of haplotypes between certain populations. For example, the presence of the A haplotype is at an average of $75 \%$ in Japanese, Han Chinese and Korean populations, but at just $13 \%$ in Australian aboriginal populations. ${ }^{24-26}$

\section{KIR and diseases}

The diversity of frequencies of the KIR and HLA haplotypes in certain populations indicates that certain individuals should have different levels of protection against certain diseases.

Analysis of mRNA and protein expression demonstrates that each NK cell clone in any given person does not express all of the KIR genes identified in their genome, but a random combination of them. Therefore, each individual has a repertoire of different NK cells with variable expression of KIR molecules on their surfaces. ${ }^{27}$

Some authors have proposed a model for classifying KIR/ HLA combinations in terms of the predicted activating or inhibitory tendencies of the NK cells. ${ }^{28,29}$ At one extreme of the spectrum are the AA haplotypes, with their inhibitory tendency, while at the other extreme are the BB haplotypes, with their inheritance of a tendency to activation. Genotypes with inhibitory receptors and deficient HLA ligands tend towards increased activation, with few NK cells under inhibitory control. This appears to be a valid model for analyzing the risk of disease, but we need more evidences for its validation. On the other hand, any model that one wishes to organize in order to understand the relationships between the KIR/HLA genotypes, must take into consideration the fact that some individuals who have truncated variants of KIR2DS4 and KIR2DL4 within the A haplotype do not express activating KIR molecules on the surface of their NK cells. Nevertheless, they apparently have normal innate immunity. In accordance with these studies, the hypothesis that at least one stimulatory receptor must exist no longer applies to NK cells. Furthermore, those NK cells that do not express inhibitory receptors 
Table 2 - Distribution of KIR genes in Brazilian Caucasoids and in other populations

\begin{tabular}{|c|c|c|c|c|c|c|c|c|c|}
\hline \multirow[b]{4}{*}{$\begin{array}{l}\text { KIR } \\
\text { Gene }\end{array}$} & \multicolumn{9}{|c|}{ Populations } \\
\hline & \multirow{2}{*}{$\frac{\text { Brazil }}{\text { Caucasian }}$} & \multicolumn{3}{|c|}{ Argentina } & \multirow{2}{*}{$\begin{array}{c}\text { UK } \\
\text { Caucasian }\end{array}$} & \multirow{2}{*}{$\begin{array}{l}\text { Australia } \\
\text { Aborigine }\end{array}$} & \multicolumn{3}{|c|}{ Mexico } \\
\hline & & Caucasian & Wichis & Chiriguanos & & & Mestizo & Purepecha & Tarahumara \\
\hline & $(n=116)$ & $(n=402)$ & $(n=101)$ & $(n=54)$ & $(n=136)$ & $(n=25)$ & $(n=24)$ & $(n=24)$ & $(n=24)$ \\
\hline 2DS2 & 60 & 55 & 61 & 41 & 51 & 85 & 44 & 34 & 34 \\
\hline 2DL2 & 66 & 56 & 62 & 44 & 49 & 79 & 43 & 34 & 34 \\
\hline 2DL3 & 86 & 87 & 84 & 87 & 92 & 67 & 100 & 100 & 100 \\
\hline 2SD3 & 34 & 29 & 3 & 6 & 24 & 81 & 17 & 4 & 0 \\
\hline 2DP1 & 96 & 96 & 84 & 91 & NT & NT & NT & NT & NT \\
\hline 2DL1 & 97 & 96 & 84 & 91 & 91 & 72 & 100 & 100 & 100 \\
\hline 3DL1 & 96 & 95 & 89 & 87 & 97 & 55 & 99 & 98 & 98 \\
\hline 3DS1 & 42 & 42 & 54 & 57 & 42 & 78 & 42 & 62 & 66 \\
\hline 2DL5 & 53 & 56 & 53 & 59 & NT & NT & 49 & 62 & 66 \\
\hline 2DS5 & 29 & 36 & 52 & 56 & 32 & NT & 40 & 62 & 66 \\
\hline 2DS1 & 36 & 46 & 53 & 57 & 45 & 82 & 42 & 62 & 66 \\
\hline 2DS4 & 96 & 95 & 89 & 87 & 96 & 51 & 98 & 98 & 98 \\
\hline
\end{tabular}

NT = not tested.

that recognize self-HLA are apparently unresponsive and relatively tolerant to autologous cells. The mechanism responsible for this effect is unknown, but it is important to point out that inhibitory receptors that recognize the HLA in their cells could have a positive role in the maturation of NK cells, giving them a license to kill. ${ }^{30}$

In summary, the balance between inhibition and activation means that the NK cell can help the body in its natural immune surveillance, even before acquired immunity participates. Infectious agents are eliminated or partially destroyed by the action of the NK cells. Furthermore, there are other pathologies in which these systems have equal importance, such as certain autoimmune diseases, tumors, preeclampsia and recurrent miscarriages. There have been suggestions that activated KIR can recognize class I HLA molecules that contain peptides related to certain pathologies or even other types of ligand that serve to identify abnormal cells.

\section{HIV}

The innate and adaptive immune systems are important during several stages of the HIV infection, including in its progression. They are positively important in defense against acute infection, and can even limit disease progression.

Natural killer cells can lyse, in vitro, target-cells infected with HIV. Defects in NK cell function can be associated with the disease progression. It is known that HIV reduces HLA expression, and this activates NK cells to lyse target-cells. Nevertheless, it is also known that the virus reduces expression of the HLA-A and B molecules, but may maintain HLA-C expression, helping it to evade recognition by the NK cells. ${ }^{31}$

Analysis of HLA-B alleles in infected individuals has demonstrated that homozygosis for HLA-Bw4, a ligand for KIR3DL1 and probably also for KIR3DS1, is associated with a slow decline in CD4+ Tlymphocyte counts, which are cells that make it possible to know the progression of infection. ${ }^{32}$ Furthermore, analysis of more than 1,000 individuals infected with HIV demonstrated that those with the KIR3DS1 genotype and a subtype of the HLA-Bw4 allele that has Ile in position 80 on the heavy chain (Bw4Ile80) deteriorate more slowly than those in whom the KIR/HLA activator combination is absent. Indeed, KIR3DS1 in the absence of this specific Bw4 is not protective. A synergistic effect between the two is needed for the beneficial effect. Nevertheless, the presence of an NK activator program appears to be of benefit in limiting or delaying onset of the disease. It has been suggested that the NK cell receptor (KIR3DS1) interacts with HLA-B Bw4-Ile80 which contains HIV peptides, activating the NK cells to eliminate the infected cell. In the same population of infected patients, it was observed that the KIR3DS1/Bw4Ile80 combination had a delayed risk of opportunistic infections. ${ }^{33}$ 
Several different studies have tried to find the reasons why some sex professionals do not become seropositive. Although they have not found a complete answer, it appears clear that the immunogenetics involved, translated into the ability to block the entrance of HIV and the resulting seroconversion, are not the same that control disease progression. In a study carried out in Abidjan, in the Ivory Coast, it was demonstrated that exposed professionals who were seronegative often exhibited inhibitory KIR genes in the absence of their cognate HLA genes, stimulating the activation of NK cells with resulting protection against disease progression. These exposed and seronegative women also had a greater frequency of KIR genotypes with more activating receptors. ${ }^{34}$

\section{Hepatitis C}

One hundred and seventy million people have the hepatitis $\mathrm{C}$ virus (HCV) and they may either be cured or become chronically infected. The second class of patients has anti-HCV antibodies and HCV-RNA in circulation and may progress to cirrhosis and hepatoma. Patients are considered cured if the viral genome cannot be detected in circulation for at least 2 years.

There is a theoretical hypothesis that the response of innate immunity due to NK cells may stimulate maturation of the dendritic cells. These, in turn, would activate the adaptive immunoresponse by T lymphocytes, making possible an effective attack against cells infected with HCV.

It has been demonstrated that the subset of patients that cure had a high frequency of homozygosis for group 1 HLA-C and of homozygosis for KIR2DL3. This allele's affinity for binding to HLA-C is less than that of KIR2DL2 or KIR2DL1 for their ligands. These findings could be related to a beneficial effect from inhibition, allowing greater chances for stimulation of activating receptors. Patients with lighter viral loads are also benefited by this combination of homozygosis. ${ }^{28}$

The results described above encourage the idea that there may be a quantitative model of NK cell programming, dependent on the functional differences between heterozygosis and homozygosis, both for the KIR genes and their HLA ligands. The increased inhibition favors activation and may benefit patients.

\section{The significance of NK cells for bone marrow transplantation}

Immunological reactivity after BMT is important. In order to reduce the chances of rejection, myeloablation is used, which is to say, destruction of the patient's bone marrow by chemotherapy. This procedure makes it possible to destroy the patient's lymphoid tissues and their leukaemic tumor cells and makes space for the transplanted marrow. Graft versus host disease (GVHD) is expected in a large proportion of cases, being caused by CD8 T lymphocytes from the donor, which, in particular, attack the intestine, skin and liver of the transplant patient. This takes place after a lymphokine "storm" resulting from the myeloablative destruction, and also dependent on HLA disparity between donor and recipient. Therefore, it would be ideal to transplant patients with $T$ lymphocyte-depleted bone marrow, reducing the risk of GVHD and any discrete class I HLA incompatibility. This small disparity or alloreactivity serves to stimulate the donor's NK cells and CD8 T lymphocytes to perform an attack against the patient's bone marrow, eliminating leukemic cells (the graft versus leukemia effect) and residual marrow tissue. Among the advantages of discrete alloreactivity are the immunological recovery, the reduction of relapses and myeloablative chemotherapy. ${ }^{35}$

When transplants are performed without graft lymphocyte depletion, the presence of activating KIR in the donor provokes immunological reaction, whereas, when present in the recipient it induces tolerance.

An increase in acute GVHD was identified with KIR2DS3 donors in unrelated HLA identical donor transplants and in cases where the donor had more than four activating KIR in haploidentical transplants.

With the intention of simplifying analyses of effectiveness, authors have grouped patients into A and B haplotypes, where the first has more inhibitory KIR genes, and the second more activating genes, and also to make it possible to observe whether HLA-C1 and C2 ligands could also be used to evaluate the best KIR-HLA compatibility for BMT in leukemia patients. ${ }^{35}$ With relation to the $A$ and $B$ haplotypes, they examined the donor-recipient relationship, considering the homozygous AA group (with the greatest number of inhibitory genes) when only genes of group A were present. All other individuals had one or more genes specific to the $B$ haplotypes. These could be AB heterozygotes or BB homozygotes, always expressing stimulatory KIR genes. Approximately 28 and $30 \%$ of donors and recipients, respectively, had the $A A$ haplotype, where all others had, at least, one $B$ haplotype. $A$ survival analysis of 202 patients indicated a discrete, and not significant tendency to lower survival among AA patients receiving BX grafts. Nevertheless, in 113 patients with myeloid leukemia, a beneficial effects was demonstrated when $B X$ patients received grafts from AA donors. In contrast, survival is 3.8 times worse for AA patients given BX grafts ( $p=$ 0.046 ). In other words, $B$ haplotypes (with more stimulatory genes) in the donor are prejudicial to this type of transplant, while their presence in the recipient may be of benefit. These results were analyzed and shown to be independent of patient age and disease stage. ${ }^{36}$

The combination of $\mathrm{BX}$ donor and $\mathrm{AA}$ recipient was considered a risk factor for disease relapse (myeloid leukemia) and acute GVHD degrees II-IV. In a group of 112 transplanted patients, the incidence of acute GVHD was $46 \%$ in AA recipients with $B X$ donors; $10 \%$ for $A A$ recipients with $A A$ donors; 
$20 \%$ for BX recipients with AA donors and $30 \%$ when both recipient and donor were $B X$.

The most dominant ligands for the KIR receptors are the HLA-C molecules. The difference between the C1 and C2 ligands is that $\mathrm{C} 2$ produces stronger inhibition than $\mathrm{C} 1$. Therefore, the same study divided the transplanted patients into the two groups in which the recipients and their HLA-identical donors were C2. Recipients without C2 alleles were defined as $\mathrm{C} 1$ homozygotes ( $\mathrm{C} 1 \mathrm{C} 1$ ). Recipients with $\mathrm{C} 2$ alleles, including $\mathrm{C} 2$ homozygotes, were defined as $\mathrm{C} 2 \mathrm{CX}$. Surprisingly, the combination of $\mathrm{BX}$ donor and $\mathrm{AA}$ recipient was only identified as a risk factor for acute GVHD in C1C1 transplants, and not in C2CX. When the effect of the HLA-C type was analyzed alone, a mild tendency to risk of GVHD was detected in C1C1 cases, but not in $\mathrm{C} 2 \mathrm{C} 2$.

In summary, the best survival rates among transplanted myeloid leukemia patients is associated with group B KIR being present in the recipient and absent in the donor. The worst survival rates are observed when the donor has group $B$ and the recipient does not. This last combination is also related with increased relapse and acute GVHD rates. However, these deleterious effects were only seen when donor and recipient were homozygotes for the ligand KIR-C1.

\section{Autoimmunity}

Several mechanisms are hypothesized to explain the participation of NK cells in autoimmunity. Inappropriate activation or lack of inhibition may be responsible for the pathogenic function with the help of active T cells. Scleroderma is a disease involving tissue fibrosis, inflammation and vascular damage. Recent study describes an association with the NK cell activators KIR2DS1 and or KIR2DS2 ${ }^{37}$. Another interesting example of this effect can be seen in psoriasis arthritis, where KIR2DS1 and/or KIR2DS2 confer a high risk of the disease when the inhibitory receptors KIR2DL1 and KIR2DL2/3 are absent. A recent proposal suggests a model of susceptibility to this disease due to the presence of homozygosis for HLA-C ligands, minimizing inhibitory signals and producing strong activation. $^{38}$

A similar situation has been identified in rheumatoid vasculitis, where risk is associated with the presence of KIR2DS2. In a similar manner, diabetes type $I$ is associated with an increase in KIR2DS2/HLA ligands in the presence of inhibitory interactions. The authors suggest a model in which the KIR function are secondary but provokes low affinity for the T lymphocytes responsible for autoimmunity. ${ }^{39}$

Psoriasis vulgar (PV) is a chronic inflammatory skin disease, the pathogenesis and genetic influence of which remain unclear. Earlier studies have suggested an association between this pathology and KIR2DS1 receptors and with HLA-C0602, where people with the second of these alleles have a 10 times greater chance of suffering from PV. In the Brazilian Caucasian population, we found the Cw0602 allele in $26.5 \%$ of the patients with PV, compared with just $5.4 \%$ of the controls ( $p<0.001)$. The KIR2DS1 did not reach statistic significance in prevalence.

With relation to the KIR2DS1 activating gene, some publications have shown that it is statistically more prevalent among PV patients than healthy controls. ${ }^{40}$ Other studies intend to demonstrate that PV is a disease in which these activating KIR receptors are present in the absence of their homologous HLA ligands. Therefore, in addition to other environmental factors, PV is the result of a balance between activating and inhibitory genotypes where activation predominates. ${ }^{37}$

\section{Conclusions}

The intention of this article was to introduce a summary of the current knowledge about innate immunity and its relationship with patients' immune surveillance. The importance of this in pediatrics can be anticipated for premature patients, newborn infants and for all of those who have not developed solid acquired immunity. The NK cells, their receptors and genes are part of maintaining good health, especially during this stage of life, when acquired immunity is not well effective. The combination of these genes and receptors are partners for pediatricians, when they prefer to wait for recovery, before given antibiotics early in the onset of an infection. It is during this period of waiting for recovery that innate mechanisms participate and, in the majority of times, they manage to overcome the infectious agents. . With this review we show the Immunogenetic bases of the KIR/HLA system that has recently been identified as of importance for the prevention of infection, in bone marrow transplants and autoimmunity. The results of the KIR genes and haplotypes frequencies in a Brazilian Caucasian control group were also presented.

\section{Acknowledgements}

We are grateful to the group of the Immunology Service at the Hospital de Clínicas de Porto Alegre.

\section{References}

1. Parham P. Taking license with natural killer cell maturation and repertoire development. Immunol Rev. 2006;214:155-60.

2. Parham P. The body's defenses against infection. In: Parham $P$, editors. The imune system. New York: Garland Science; 2005. p. 252-6.

3. Vilches C, Parham P. KIR: diverse, rapidly evolving receptors of innate and adaptative immunity. Annu Rev Immunol. 2002;20: 217-51.

4. Ljunggren HG, Pääbo S, Cochet M, Kling G, Kourilsky P, Kärre K. Molecular analysis of $\mathrm{H}-2$ deficient lymphoma lines. Distinct defects in biosynthesis and association of MHC class I heavy chains and beta 2-microglobulin observed in cells with increased sensitivity to NK cell lysis. J Immunol. 1989;142:2911-7. 
5. Ljunggren HG, Karre K. In search of the "missing self": MHC molecules and NK cell recognition. Immunol Today. 1990;11: 237-44.

6. Boyton RJ, Altmann DM. Natural killer cells, killer immunoglobulin-like receptors and human leucocyte antigen class I in disease. Clin Exp Immunol. 2007;149:1-8.

7. Degli-Esposti MA, Smyth MJ. Close encounters of different kinds: dendritic cells and NK cells take centre stage. Nat Rev Immunol. 2005;5:112-24.

8. O'Connor GM, Hart OM, Gardiner CM. Putting the natural killer cell in its place. Immunology. 2006;117:1-10.

9. The Immuno Polimorphism Database. KIR database. http:// www.ebi.ac.uk/ipd/kir

10. André $\mathrm{P}$, Biassoni R, Colonna M, Cosman D, Lanier LL, Long EO, et al. New nomenclature for MHC receptors. Nat Immunol. 2001; 2:661.

11. Cook MA, Norman PJ, Curran MD, Maxwell LD, Briggs DC, Middleton $\mathrm{D}$, et al. A multi-laboratory characterization of the KIR genotypes of 10 th International Histocompatibility Workshop cell lines. Hum Immunol. 2003;64:567-71.

12. Hsu KC, Chida S, Geragthty DE, Dupont, B. Killer cell immunoglobulin-like receptor (KIR) genomic region: gene-order, haplotypes and allelic polymorphism. Immunol Rev. 2002;190: 40-52.

13. Hsu KC, Liu XR, Selvakumar A, Mickelson E, O'Reilly RJ, Dupont B. Killer Ig-like receptor haplotype analysis by gene content: evidence for genomic diversity with a minimum of six basic framework haplotypes, each with multiple subsets. J Immunol. 2002;169:5118-29.

14. Shilling HG, Guethlein LA, Cheng NW, Gardiner CM, Rodriguez $R$, Tyan $D$, et al. Allelic polymorphism synergizes with variable gene content to individualize human KIR genotype. J Immunol. 2002;168:239-47.

15. Biassoni R, Falco M, Cambiaggi A, Costa P, Verdiani S, Pende D, et al. Amino acid substitutions can influence the natural killer (NK)-mediated recognition of HLA-C molecules. Role of serine-77 and lysine- 80 in the target cell protection from lysis mediated by "group2" or "group 1"NK clones. J Exp Med. 1995;182:605-9.

16. Cella M, Longo A, Ferrara GB, Strominger JL, Colonna M. NK3-specific natural killer cells are selectively inhibited by Bw4-positive HLA alleles with isoleucina 80. J Exp Med. 1994; 180:1235-42.

17. Miller SA, Dykes DD, Polesky HF. A simple salting out procedure for extracting DNA from human nucleated cells. Nucleic Acids Res. $1988 ; 16: 1215$.

18. Gomez-Lozano N, Vilches C. Genotyping of human killer-cell immunoglobulin-like receptor genes by polymerase chain reaction with sequence-specific primers: an update. Tissue Antigens. 2002:59:184-93.

19. Bunce M, O'Neill CM, Barnardo MC, Krausa P, Browning MJ, Morris $P J$, et al. Phototyping: comprehensive DNA typing for HLA-A, B, C, DRB1, DRB3, DRB4, DRB5 \& DQB1 by PCR with 144 primers mixes utilizing sequence-specific primers (PCR-SSP). Tissue Antigens. 1995;46:355-67.

20. Contreras G, Aláez C, Murguía A, Garcia D, Flores H, Gorodezky C. Distribution of the killer cell immunoglobulin-like receptors in Mexican Mestizos. Tissue Antigens. 2007;69 Suppl 1:125-9.

21. Flores, AC, Marcos CY, Paladino N, Capucchio M, Theiler G, Arruvito $L$, et al. KIR genes polymorphism in Argentinean Caucasoid and Amerindian populations. Tissue Antigens. 2007: 568-76.
22. Gutiérrez-Rodríguez ME, Sandoval-Ramírez L, Díaz-Flores $M$, Marsh SG, Valladares-Salgado A, Madrigal JA, et al. KIR gene in ethnic and mestizo population from Mexico. Hum Immunol. 2006,67:85-93

23. Norman PJ, Stephens HA, Verity DH, Chandanayingyong D, Vaughan RW. Distribution of natural killer cell immunoglobulin-like receptor sequences in three ethnics groups. Immunogenetics. 2001;52:195-205.

24. Jiang K, Zhu FM, Lv QF, Yan LX. Distribution of killer cell immunoglobulin-like receptor genes in the Chinese Han population. Tissue Antigens. 2005;65:556-63.

25. Yawata M, Yawata N, McQueen KL, Cheng NW, Guethlein LA, Rajalingam R, et al. Predominance of group A KIR haplotypes in Japanese associated with diverse NK cell repertoires of KIR expression. Immunogenetics. 2002;54:543-50.

26. Toneva M, Lepage V, Lafay G, Dulphy N, Busson M, Lester S, et al. Genomic diversity of natural killer cell receptor genes in three populations. Tissue Antigens. 2001;57:358-62.

27. Valiante NM, Uhrberg M, Shilling HG, Lienert-Weidenbach $K$, Arnett KL, D'Andrea A, et al. Functionally and structurally distinct NK cell receptor repertoires in the peripheral blood of two human donors. Immunity. 1997; 7:739-51.

28. Khakoo SI, Carrington M. KIR and disease: a model system or system of models. Immunol Rev. 2006;214:186-201.

29. Williams AP, Bateman AR, Khakoo, SI. Hanging in the balance: KIR and their role in disease. Mol Interv. 2005;5:226-40.

30. Kim S, Poursine-Laurent J, Truscott SM, Lybarger L, Song YJ, Yang $L$, et al. Licensing of natural killer cells by host major histocompatibility complex class I molecules. Nature. 2005;436: 709-13.

31. Flores-Villanueva PO, Yunis EJ, Delgado JC, Vittinghoff E, Buchbinder S, Leung JY, et al. Control of HIV-1 viremia and protection from AIDS are associated with HLA-Bw4 homozygosity. Proc Natl Acad Sci USA. 2001;98:5140-5.

32. Martin MP, Gao X, Lee JH, Nelson GW, Detels R, Goedert JJ, et al. Epistatic interaction between KIR3DS1 and HLA-B delays the progression to AIDS. Nat Genet. 2002;31:429-34.

33. Jennes W, Verheyden S, Demanet C, Adjé-Touré C, Vuylsteke B, Nkengasong JN, et al. Cutting edge: resistance to HIV-1 infection among African female sex workers is associated with inhibitory KIR in the absence of their HLA ligands. J Immunol. 2006;177: 6588-92.

34. Ruggeri L, Capanni M, Urbani E, Perruccio K, Shlomchik WD, Tosti $A$, et al. Effectiveness of donor natural killer cell alloreactivity in mismatched hematopoietic transplants. Science. 2002;295:2097-100.

35. McQueen KL, Dorighi KM, Guethlein LA, Wong R, Sanjanwala B, Parham P. Donor-recipient combinations of group A and B KIR haplotypes and HLA class I ligand affect the outcome of HLA-matched, sibling donor hematopoietic cell transplantation. Hum Immunol. 2007;68:309-23.

36. Momot $\mathrm{T}$, Koch S, Hunzelmann N, Krieg T, Ulbricht $\mathrm{K}$, Schmidt RE, et al. Association of killer cell immunoglobulin-like receptors with scleroderma. Arthritis Rheum. 2004;50:1561-5.

37. Pellett F, Siannis F, Vukin I, Urowitz MB, Gladman D. KIRs and autoimmune disease: studies in systemic lupus erythematosus and scleroderma. Tissue Antigens. 2007; 69: 106-108.

38. Nelson GW, Martin MP, Gladman D, Wade J, Trowsdale J, Carrington M. Cutting edge: heterozygote advantage in autoimmune disease: hierarchy of protection/susceptibility conferred by HLA and killer Ig-like receptor combinations in psoriatic arthritis. J Immunol. 2004;173:4273-6. 
39. van der Slik AR, Alizadeh BZ, Koeleman BP, Roep BO, Giphart MJ. Modelling KIR-HLA genotype disparities in type I diabetes. Tissue Antigens. 2007;69:101-5.

40. Bashirova AA, Martin MP, McVicar DW, Carrington M. The killer immunoglobulin-like receptor gene cluster: tuning the genome for defense. Annu Rev Genomics Hum Genet. 2006; 7:277-300.
Correspondence:

Luiz Fernando Jobim

Serviço de Imunologia - Hospital de Clínicas de Porto Alegre

Rua Ramiro Barcelos, 2350

CEP 90035-903 - Porto Alegre, RS - Brazil

E-mail: ljobim@hcpa.ufrgs.br 